\title{
Images of Sonic Objects
}

\author{
ROLF INGE GODØY \\ Department of Musicology, University of Oslo, P.B. 1017 Blindern, N-0315 Oslo, Norway \\ E-mail: r.i.godoy@imv.uio.no
}

\begin{abstract}
Based on innumerable informal accounts and a number of scientific studies, there can be no doubt that people often have quite vivid images of musical sound in their minds, and that this is the case regardless of levels of musical training. Various introspective accounts and more recent neurocognitive research seem to converge in suggesting that imagery for music is closely linked with imagery for music-related actions. In this paper, the consequences of sound-action links for our notions of the sonic image are discussed, with a particular focus on the relationship between sonic objects and action chunks. In conclusion, the exploitation of action imagery is seen as holding great promise in enhancing our means for musical imagery in various creative, research and educational contexts.
\end{abstract}

\section{INTRODUCTION}

Regardless of levels of musical training, most people seem to have memory images of music, and also seem to be able to give verbal accounts of these images, of what we in our context call sonic images. Given this seeming universality of sonic images, we are faced with two obvious but non-trivial issues.

- Music is an ephemeral phenomenon in the sense that at one moment the sound is here, the next moment it is gone. Apparently, we are somehow able to create more or less solid images in our minds of the sound, and we are often able to conjure up some image of what the music sounded like also long after we heard it. This concerns not only various issues of memory and recall (Snyder 2000), but also of perception and transformation, or re-coding and chunking, of continuous sound into somehow stable entities in our minds (Godøy 2008).

- Musical sound will usually appear as feature-rich or multidimensional, and although we can try to point to features in the sound in playback or performance, or try to display acoustic and/or perceptual data associated with the sound, we often make use of various verbal metaphors when communicating with other people about our sonic images. Such verbal metaphors are very useful both in practical work (e.g. Porcello 2004) and in research (e.g. Kendall and Carterette 1993), yet we have challenges in trying to give more precise meanings to these metaphors.

The emergence and nature of sonic images concerns various disciplines such as acoustics, psychoacoustics, the cognitive sciences and philosophy, as well musicology and other music-related disciplines. Sonic images may often be multimodal as well as have rich sociocultural significations, and this means that there may be several different and/or complementary approaches to the study of sonic images. The approach in this paper will be by way of sonic objects based on the theories of Pierre Schaeffer and his co-workers (Schaeffer 1966, 1998) as well as some more recent extensions (summarised in Godøy 1997). Briefly stated, sonic objects are holistically perceived fragments of sound, typically with durations in the 0.5 to 5 seconds range. It will be argued that sonic objects constitute the most significant timescale of music in relation to the study of sonic images. Sonic objects are at the nexus of several musical and cognitive constraints, allowing us to study closely the emergence and depictions of stable images from continuous sound. The aim of this paper is then to give an overview of the various elements that go into shaping images of sonic objects.

In the next sections, sonic objects are first seen in the context of musical imagery, something that subsequently may be understood in the perspective of embodied music cognition. With this background, we shall have a look at different timescales for the manifestation of various perceptually salient features of music as the basis for sonic objects and soundrelated actions, something that leads us to the idea of action imagery in musical imagery, an idea that can have various applications in musical practice and research.

\section{MUSICAL IMAGERY}

The term 'musical imagery' may have many different meanings, ranging from denoting images of the acoustic signal to various images associated with the performance, the perception, and the emotive experience of the music, extending also to more extramusical image associations that listeners may have to musical sound, such as images of landscapes or historical events. Aware of this multitude of possible significations, we defined musical imagery in the call for papers prior to an international conference on musical imagery in 1999 as 'our mental capacity for imagining musical sound in the absence of a directly audible sound source, meaning that we can recall and 
re-experience or even invent new musical sound through our "inner ear", (Godøy and Jørgensen 2001: ix). This may be a useful initial definition, but there is of course much more to musical imagery, both with regards to the content or nature of images and with regards to the workings or phenomenal underpinnings.

Musical imagery may be seen in the broader context of what is generally referred to as mental imagery, a term denoting our capacity for having internal images of the world, as somehow originating in, but not necessarily truthfully reflecting external experience, because bits and pieces from lived experience may be recombined in novel and/or fictional ways. Mental imagery has a history in Western philosophy extending back to antiquity (see Schneider and Godøy 2001 for an overview), but in the 1980s and 1990s has been more systematically studied, and has been the topic of the so-called 'mental imagery debate' (see e.g. Kosslyn 1994 for an overview). One main element in this debate was the view of mental imagery as strongly linked to the external world, or to what we could call having a strong ecological content, versus the view of mental imagery as more abstract, as a so-called 'symbol manipulating' phenomenon more in line with the workings of some kind of computation machine. Another main element was the emergence of so-called 'functional equivalence' between real-world perception and action, and imagined perception and action. This meant that acts of imagery showed similarities with real world acts, e.g. that mentally rotating a figure would take more time if the rotation was more extensive than if it was just a small tilting, or that recalling the text of the last verse of a song would take a longer time than the first verse, as people often would scan through the whole song from the beginning. With enhanced means for brain activity observations, there emerged evidence that mental imagery makes use of much of the same neurophysiological apparatus as real perception and action. Both the behavioural functional equivalence and the shared neurophysiological apparatus of mental imagery and real-world perception and action are important for our present understanding of musical imagery. Many features of real-world music perception and music-related actions extend into the realm of musical imagery and can thus serve as points of departure for exploring sonic images.

Although visual domain mental imagery has the longest research record (see Kosslyn 1994 for an overview), there has previously also been some studies of auditory imagery (Reisberg 1992), as well as our own collection of studies of musical imagery (Godøy and Jørgensen 2001). However, the profusion of non-invasive brain observation methods since 2000 has clearly strengthened both the ecological and the functional equivalence aspects of mental imagery, including musical imagery. Of particular interest is the emerging evidence for links between sonic imagery and movement imagery (see Zatorre and Halpern 2005 for an overview). It has, for instance, been shown that when professional pianists listen to piano music, there is an activation of the motor control apparatus in the brain as if they themselves were playing piano (Haueisen and Knösche 2001), and this coupling of piano sound with sound-producing movement can also be learned in a matter of weeks of piano training (Bangert and Altenmüller 2003). The links between sound and movement images may be partly hard-wired (Kohler, Keysers, Umiltà, Fogassi, Gallese and Rizzolatti 2002), and there is now also increasing support for the so-called 'motor theory' of perception, a theory that for decades has suggested that perception is indeed closely linked with mental simulation of sound-producing actions (Galantucci, Fowler and Turvey 2006). In our context it is significant to note that there is a bidirectionality between images of sound and action - for example, that just seeing silent piano performance actions may invoke mental images of sound (as suggested by Haslinger, Erhard, Altenmüller, Schroeder, Boecker and Ceballos-Baumann 2005) - and this may perhaps hold the key to a more volitional element of musical imagery: if you want to create a mental image of the sound, imagine that you are producing the sound or making some movement to the sound.

Neurocognitive studies may give us valuable information about the workings of musical imagery and, importantly, strengthen the links between musical imagery and motor imagery, but we still need other approaches as well to tell us more about the content of musical imagery. One possible approach is then to observe how people move to music, and to try to deduce something about covert mental sonic images from overt behaviour.

\section{EMBODIED MUSIC COGNITION}

Since the end of the 1990s there has been a deluge of publications on so-called 'embodied cognition'. Although this is a rather heterogenous topic, the common element for our context is that of seeing human mental activity as intimately linked with sensations of movement. The basic idea is that whatever we perceive or think is correlated with mental simulations of body movement, both those of other people (Wilson and Knoblich 2005) and of our own prospective movements (Gallese and Metzinger 2003), and with simulations of what we believe are the cause, and even tactile, kinematic features of what we perceive (Berthoz 1997).

Keeping in mind that musical sound has traditionally been made by body movement, and seeing how people make various kinds of body movement to music in listening situations, as well as thinking of the 
countless verbal reports people make about movement sensations in musical experience, we have come to believe that images of body movements are integral to musical experience (Godøy 2003; Leman 2008; Godøy and Leman 2010). We also believe that association of body movement with music is universal and independent of levels of musical training, because most people have extensive experience of the links between sound and movement. This is evident from various instances of so-called 'air instrument' performance such as air guitar, air drums and air piano, where even people with no musical training seem to have some knowledge of how the sounds are produced - that is, of the workings and spatial layout of musical instruments, and of the kinematics as well as the dynamics of sound-producing gestures (Godøy, Haga and Jensenius 2006a). Also, there seemed to be a fair amount of agreement in the spontaneous drawings of gestures that people with different levels of musical training made to musical excerpts in socalled 'sound tracing' studies, as long as the musical excerpts did not have too many features; for example, there was good agreement for pitch contours and salient dynamic patterns, but much less agreement for multidimensional timbral features (Godøy, Haga and Jensenius 2006b; Haga 2008). Similarly, there seemed to be a fair level of consensus in the spontaneous gestures that dancers made to music with regard to the overall patterns of movement and sense of effort, although the details varied (Haga 2008).

These and other studies (e.g. Eitan and Granot 2006) support the idea that some sense of movement, and hence also kinematics or movement shape, is often spontaneously associated with music. We may speak of motormimetic sketching of musical sound, meaning that there is a continuum between very vague, low-acuity gestural images of musical sound and more precise, high-acuity gestural images of musical sound, dependent upon the level of expertise of the listener (Godøy et al. 2006a). Actually, this variable acuity is a phenomenon built into the music itself in that a musical passage, a rapidly ascending scale on the piano for example, may be perceived with high acuity as a series of individual tone-events associated with individual finger-events, but may also be perceived with low acuity at a more superordinate level as an ascending contour of sound (individual tones fused into a chunk or gestalt) coupled with a similar fusion of the finger actions into an ascending gesture of the wrist, elbow, and maybe even shoulder and torso. The point is that all toneevents or sound-events in this embodied perspective are understood as included in some kind of gesture trajectory, and that images of these gesture trajectories can be efficiently transmitted by musical sound to the listener, who in turn may spontaneously perceive these trajectories because of massive past experience of soundgesture links. Yet with increasingly complex musical textures, it is also evident that musical sound can convey several different gestures-inducing cues; for example, in a musical excerpt containing a highly embellished foreground melody, plus an energetic drum accompaniment, and a protracted string background, the listener may selectively focus on any of these textural features, hence we may speak of rich gestural affordances of musical sound (Godøy 2010a).

The awareness of sound-producing gestures is a challenge to traditional Western ideas of music theory in that the sound-producing gestures may be seen as primordial to the tone: the sound-producing gesture starts before, and often continues after, the tone/sound, hence may be seen as a precondition for music. Also, thinking sound-producing gestures as integral to music may help us to bridge the gaps between the different levels in music: the gesture is at the one and same time both concerned with the micro-level of the singular tone/sound and the fusion of several tones/sounds into higher-level units. This fusion into more superordinate chunks may be regarded as an instance of coarticulation, meaning that otherwise individual events and actions are contextually smeared and subsumed into some higher-level chunk (Godøy 2008; Godøy, Jensenius and Nymoen 2009). In the case of the rapidly ascending scale on the piano, the fusion of individual tone-events and tone-onset finger actions into a higherlevel ascending sound contour and movement contour is a typical case of coarticulation. Notably, then, coarticulation in our context (as well as in linguistics and other contexts) concern both the production and the perception of musical sound.

It seems reasonable to suggest that most features of musical sound may somehow be related to body movement, including movements of our vocal apparatus, as can for instance be seen in cases of so-called scat singing and beatboxing, where there seems hardly to be any limit to the possibilities of vocal imitation of sounds. We should also take into account the phenomenon of so-called motor equivalence (Kelso, Fuchs, Lancaster, Holroyd, Cheyne and Weinberg 1998), meaning that one effector may be substituted for another effector (e.g. that the vocal apparatus can imitate sounds that are non-vocal in origin, or that we can try to trace with our hands the shape of a sound that is produced by other instrumental or electronic means). The essential element of this is the motor involvement, be that mental or overt, with what we perceive and think. Notably, this also goes for visual images as suggested in Berthoz 1997, hence that seeing some kind of graphical representation of sound may also induce a sensation of sound-related movement in our minds. In summary, the basic tenet of embodied cognition is that of constant mental (as well as sometimes also overt) simulation of what we hear and see, something that we can exploit in developing our sonic images. 


\section{TIMESCALES AND FEATURES}

Music-related body movements, as well as musical sound, may be observed at different timescales: at more macroscopic timescales in view of features such as overall mode of movement and sound in a musical work (e.g. as calm, agitated, fast or slow), or at a more local timescale of singular gestures and singular sound events (e.g. of local contours, or rhythmical, textural or timbral features). In actual musical experience, we may assume that both musical sound and music-related movement are working at different timescales in parallel - in other words, that we hear the local in the context of the global and, conversely, that we experience the global as a result of concatenations of local sonic events.

With the well-known timescale for perceiving pitch and some timbral features (i.e. the stationary spectral features) extending roughly from 20 to 20,000 events per second, the timescale of less than 20 events per second is where we find various textural and rhythmical features of sound such as the Schaefferian typological categories and their corresponding body movements (more on this later). As for the 20 to $20,000 \mathrm{hz}$ timescale, pitch and stationary timbral features may of course be correlated with body movements, like in singing, humming or whistling a tone, or like in making different vowel shapes of the vocal tract for different quasi-stationary timbres.

In addition to such an event-density timescale, we also have thresholds for how long a segment of sound we need to hear in order to perceive certain features, meaning that we also have minimum duration thresholds for musical features. On the microlevel, there are for instance duration thresholds for perceiving pitch, some timbral features, event simultaneity and event order (see Moore 1995 for an overview). But for longer timescales (i.e. those of various rhythmic, textural and timbral patterns), we seem to lack more systematic studies. One of the few studies we know of in this area seems to suggest that minimum duration thresholds may sometimes be as short as 250 milliseconds for recognising salient stylistic features (Gjerdingen and Perrott 2008), and our own informal tests seem to indicate that listeners' minimum duration thresholds may vary considerably from several seconds down to less than 500 milliseconds for perceiving salient musical features, in other words seem to be within the approximate duration limits for Schaeffer-inspired sonic objects.

Although the focus on the sonic object initially was a pragmatic necessity in the early days of the musique concrète as a 'closed groove' (sillon fermé) on the phonogram disc for mixing sounds, and thus has a certain flair of a serendipitous discovery, Schaeffer's focus on the sonic object is perhaps one of his most significant achievements, and in the context of this paper also one of the most significant ideas with regards to the sonic image. From our present-day perspective, we can see the following arguments in favour of a Schaefferian focus on the timescale of the sonic object.

- Style. Very short excerpts of musical sound will in many cases suffice to recognise different musical styles (Gjerdingen and Perrott 2008), and it has also been shown that fragments from more largescale musical works may be rearranged in different sequences and still preserve the overall experience of style and aesthetic well-formedness (Eitan and Granot 2008), something that should make us a bit sceptical of received Western notions of the importance of large-scale forms in music.

- Rhythm. The typical minimum duration thresholds for recognising or determining salient rhythmical patterns such as in various dance forms, musical metre, ostinatos, and so forth seem to conform with the approximate sonic object timescale of 0.5 to 5 seconds.

- Texture/timbre. As is the case for rhythmical patterns, various textural patterns in instrumental, vocal or electroacoustic music (various patterns of fluctuations, foreground-background movements, etc.), as well as various timbre-related fluctuations and transient patterns, are all within the timescale of the sonic object.

- Modal/tonal features. The timescale of the sonic object seems sufficient to determine the modal features of a group of successive tones, and partly also to form some sensation of tonal properties, e.g. tonal, functional tonal, free tonal, dodecaphonic, serial, and so on.

- Expressivity. Linked with rhythmical and textural patterns, the timescale of the sonic object seems also to be the timescale for rubato, 'feel'/'groove' or various other expressive and articulatory features.

- Body movement. It has been shown that the grand average duration for human actions is in the 3 seconds range, but also shorter or longer actions occur (Schleidt and Kien 1997) to fit with the approximate timescale of the sonic object.

With these arguments in favour of the primacy of the sonic object, we should in our context of the sonic image see the sonic object as situated in a three-level model of timescales in music:

- Micro timescale, meaning continuous sound within the sonic object.

- Meso timescale, meaning that of the sonic object or the sound and action chunks.

- Macro timescale, meaning that of larger-scale forms such as whole sections, movements, and whole works, consisting of the concatenation of successive sonic objects. 
Within the meso-level timescale of the sonic object, we have Schaeffer's so-called typological categories for denoting the overall dynamic shapes of sonic objects: the impulsive types, meaning sonic objects that have a fairly abrupt attack followed by a shorter or longer decay phase such as in percussive or plucked sounds, the sustained types, meaning sonic objects that have a more flat overall dynamical envelope such as in bowed or blown sounds, and the iterative types, meaning sonic objects that have a rapid series of sound onsets or fluctuations, such as in a tremolo or a drum roll. One essential element in our context is that these typological categories of sonic objects also correlate well with the distinct categories of impulsive, sustained and iterative body gestures (see Godøy 2006 for more on this). However, the differences between these types also have to do with timescales. If sustained sonic objects become very short, they will become impulsive sonic objects, and, conversely, if impulsive sonic objects become too long, they will become sustained. If iterative sonic objects slow down, they may become a series of independent impulsive or even sustained sonic objects. If sustained sonic objects have too much internal movement, for example too large and too rapid fluctuation in amplitude and/or harmonic content, they may become iterative sonic objects. These and various other time-dependent thresholds that we find for musical features resemble what are known as 'phase transitions' in human (and animal) movement (Haken, Kelso and Bunz 1985), meaning that incremental or linear changes may bring about sudden categorical changes, as for example the phase transition from conducting three beats per bar to one beat per bar when gradually increasing the tempo in $\frac{3}{4}$ time from slow to fast.

In addition, there are also various other envelopes such as for timbral and pitch-related features within the sonic objects that also may be mapped to various body movements at different timescales. Going through Schaeffer's theoretical apparatus, it seems that most (or perhaps all) features at different timescales may be correlated with some kind of body movement and/or posture, hence that musical textures, be they instrumental, vocal or electroacoustic, may also be seen as choreographies of sound-related movements (Godøy 2006).

\section{SONIC OBJECTS}

One essential feature of the meso-level timescale is that the sonic object can be perceived and imagined as a whole, 'all-at-once' or 'in a now'. Ideas of such instantaneous, holistic overview images have been suggested by (among others) Xenakis with his ideas of diminuition by distantiated perspectives on musical events and his notions of hors-temps, or outside time (Xenakis 1992). However, an intriguing account of such instantaneous overview images can be found in Husserl's writing on temporal consciousness (Husserl 1991), some of which dates back to 1893 . One of Husserl's main ideas was that perception and imagery proceed by a series of so-called 'now-points', where each such now-point contains a subjective sensation of the present moment, and that each such now-point includes sensations of what has just passed and, notably so, also of what is expected to come in the immediate future. Husserl claimed that perception and imagery proceed in such a point-by-point manner because in order to make sense of the world we have to take extended segments of time and sound into account: for example, in order to perceive or imagine a melody we have to have several successive tones in consciousness simultaneously, or in order to perceive a single tone we have to have several successive phases of the tone in consciousness simultaneously. There are several intriguing issues with this (see Godøy 2010b for a discussion); however, the most remarkable element in Husserl's argument is demonstrating that there has necessarily to be some kind of moment-by-moment or chunk-by-chunk mode of perception and imagery, otherwise there would only be an indistinct continuous stream of sensations.

Related ideas of the sense of the present moment or the chunking of experience has been presented by other thinkers (e.g. Michon 1978; Stern 2004), but also, in theories of memory, there are various elements in favour of chunks at the meso-level timescale, such as models of echoic and working memory (Snyder 2000). Also the concept of chunking has received much attention since the seminal work by G. A. Miller. One of Miller's main ideas was that in order to efficiently cope with the complexity of the world, we somehow have to make compressed 'recodings' of sensory information into more easily tractable and remembered units, hence the idea of chunking in perception and memory (Miller 1956).

In addition, similar ideas of chunking have been suggested to be at work in the planning and control of action (Lashley 1951). Although there has been considerable controversy as to the degree of pre-planning or anticipatory chunking (Elliott, Helsen and Chua 2001), there seems now to emerge evidence in favour of the view of action as dependent on pre-planning for efficiency, and that there is always an element of chunking and coarticulation (see section 3 above) at work both in perception and action (Rosenbaum, Cohen, Jax, Weiss and van der Wel 2007).

Provided that we agree with the various arguments in favour of the meso-level timescale of the sonic object as the most significant in music as well as in musicrelated movement, the next question becomes what are the nature and status of the sonic object. Through a series of what are really negative determinants, 
Schaeffer narrows down what the sonic object is not (here quoted from Chion 1983: 34-5):

The sound object is not the sounding body.

The sound object is not the physical signal.

The sound object is not a fragment of a recording.

The sound object is not a symbol notated in a score.

The sound object is not a state of the soul.

So what is the sonic object then? Schaeffer's answer is that it is an 'intentional unit', meaning that it is a mental image of a chunk where we make a synthesis of various features, both those occurring in parallel and those occurring sequentially, and think of it as a coherent entity (Schaeffer 1966: 263). This also touches on the often discussed issue of so-called 'reduced listening' in Schaeffer's theory: the main point of this strategy is that of primarily focusing on the rich set of features that make up the sonic object, and not really that of cutting off all links to the external world. This is something that becomes quite clear if we read closely Schaeffer's explanation of the various typological and morphological features that go into sonic objects. Real-world phenomena such as envelopes and spectral components are present, and, what is more, they are bundled into subjectively perceived salient feature categories that we may also associate with action chunks.

The links between the sonic object and the external world are also present in the criteria for the constitution of the sonic objects. Sonic objects may be artificially created by arbitrary cuttings (by the phonograph loop, the magnetic tape, or the digital sound file), but Schaeffer sought to find a more 'natural' basis for the sonic objects in what we would call qualitative discontinuities in the continuous acoustic signal. That was done with the so-called 'stressarticulation' distinction - what we could call the attack-sustain distinction. This scheme does, however, have the problem that streams of very short sounds (i.e. streams of multiple attacks) may sometimes be perceived as a unit, hence Schaeffer also applies the criteria of the overall envelopes associated with sound-producing gestures (compare this with the abovementioned typological categories of sustained, impulsive and iterative sounds). These overall dynamic envelopes of sonic objects are combined with three overall pitch-related classes for sonic objects - in other words, tonal (or pitched), non-tonal (inharmonic or noise-dominated) and variable in tonal or non-tonal content. These dynamic and pitchrelated typological classes are then combined in a $3 \times 3$ matrix, making up the very basic scheme for the typology of sonic objects. Instructive examples of this are presented in Schaeffer 1998 (CD3), and from these examples as well as Schaeffer's text we may get a quite clear sense of the action-related nature of the typological categories.
After this first differentiation of sonic objects in the typology, the morphology of sonic objects provides various progressively finer differentiations of the more internal features of the sonic object, such as the harmonic content and various patterns and evolutions in the course of sonic objects. All these feature differentiations are highly relevant for enhancing our sonic images in any kind of music, be that instrumental or electroacoustic, or based on synthetic or concrete sound, processed or unprocessed sound. Since they are universally applicable, we could thus speak of sonic object categories as generic, as may be seen from the example of iterative sounds given on tracks 25, 26 and 27 on CD3 of Schaeffer 1998. We hear in succession the sonic objects of a drum roll, a deep bassoon tone, and a fragment of electroacoustic music, hence three sonic objects with completely different origins, yet having the common feature of being iterative sonic objects.

This generic classification scheme also applies to the distinction of more complex sonic objects, to the socalled composed and composite sonic objects. The 'composed' sonic object consists of the superposition of two sonic objects, sometimes used to reinforce the attack both in instrumental and in electroacoustic music, as can be seen in figure 1. Here we have such a reinforced attack from the first movement of Lutosławski's Jeux Venitiens, and the spectrogram of Schaeffer's example of such a 'composed' sonic object from track 2 on CD3 (Schaeffer 1998). What Schaeffer called the 'composite' sonic object is made up of two sonic objects in succession that fuse into one sonic object, what we may regard as a case of coarticulation.

Again, this is a generic concept, equally well applicable to instrumental and electroacoustic music. In figure 2 we see such a coarticulated sonic object from Messian's Regard de la Vierge where a rush of demisemiquavers and the final quavers fuse together to one gesture, and next to it, a coarticulated sonic object from track 4 on CD3 of Schaeffer 1998, where we have a crescendo to an accent-point that similarly fuse.

In sum, the notion of the sonic object is based on a holistic perception and imagery of a fragment of musical sound; is generic or universal in its application; is independent of particular instrumental, vocal or electronic instantiation; and may also be correlated with some action image.

\section{ACTION IMAGERY IN MUSICAL IMAGERY}

Seeing the intimate links between sonic objects and sound-related actions, it may be argued that there is a component of action imagery in musical imagery. This means that images of sound may trigger images of actions, and, conversely, that images of actions may trigger images of sound in our minds. 


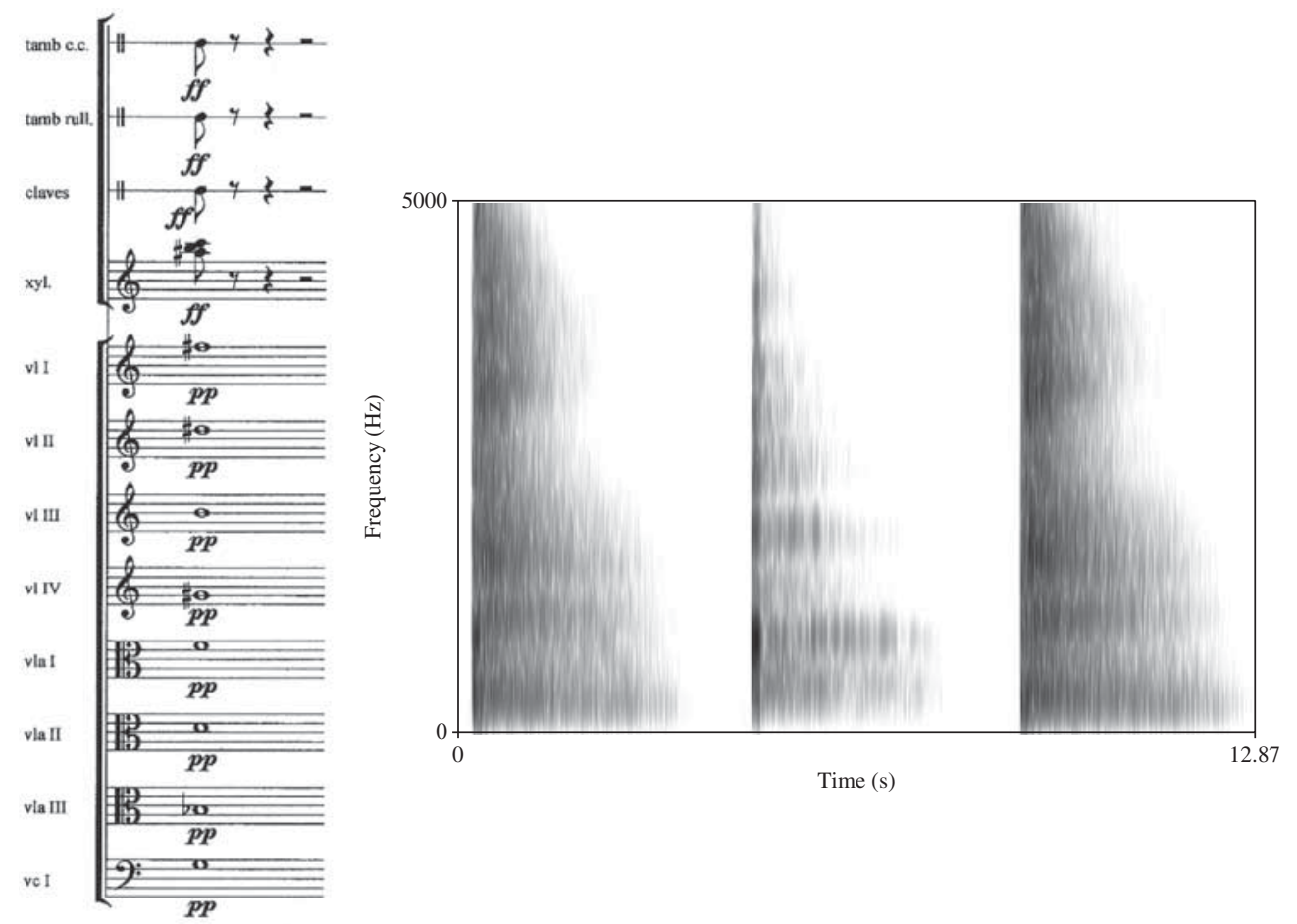

Figure 1. A 'composed' sonic object with a reinforced attack from the first movement of Lutosławski's Jeux Venitiennes, rehearsal mark F (left), and the spectrogram of Schaeffer's example of such a 'composed' sonic object with a reinforced attack from track 2 on CD3 (Schaeffer 1998), where we first see the two components separately, then together (right).
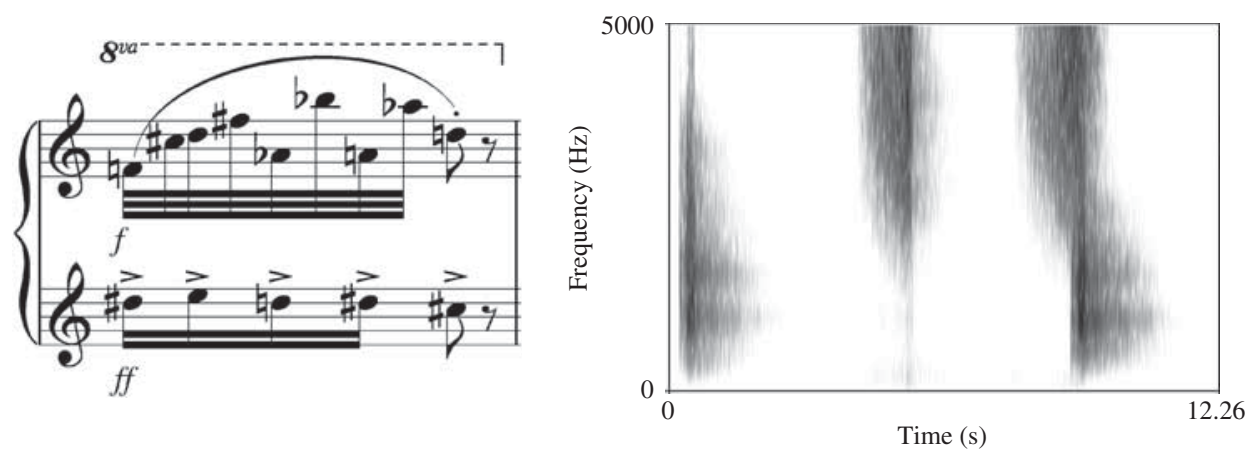

Figure 2. A coarticulated sonic object from Messian's Regard de la Vierge, bar 61, of a gesture to an accent-point (left), and next to it (right), a coarticulated sonic object from track 4 on CD3 (Schaeffer 1998), where we have a similar gesture to an accent-point, first displaying the two components and then the coarticulatory fused sonic object.

As integral elements of the sonic object, we can then think of the following music-related actions as possible active ingredients in musical imagery.

- Excitatory and modulatory actions, both of singular tones/sounds and of more composite and/or coarticulated chunks of tones/sounds. This also includes the timbre-texture continuum - that is, the continuum between sustained, iterative and impulsive sounds, and other related feature dimensions.

- Sound-tracing actions, meaning actions that are not directly related to sound-production but still closely related to sound features such as tracing (by hand, or by other body movement) the resonant envelope, spectral shape, and so on of a sonic object.

- Sound-accompanying actions, meaning various actions that are somehow in synchrony with some features of the music, e.g. as in dance and other body movement to music, but where the kinematics or the geometry of the action trajectories may be more independent of the music.

The kinematics and dynamics of sound-related actions can create images of sonic objects, visualising and making tangible the otherwise ephemeral sound. This has potentially a number of applications in 
composition, improvisation, musical performance, music education and rehabilitation, musicology and music information retrieval, as well as in music technology. For instance, the relationship between action and sound concerns various interface developments in general, and the subject of mapping in particular (e.g. Wanderley and Battier 2000; Hunt, Wanderley and Paradis 2003). Such action-sound links also have potential applications in research, and we are presently developing hand-held devices equipped with various sensors (i.e. accelerometers, and pressure and position sensors) that we hope will allow us to navigate in multidimensional sonic feature spaces by actions, hence exploiting the potential of embodied images of sonic objects in various contexts. In all this work, the many links between actions and sonic objects will be our guiding principle.

\section{CONCLUSIONS}

Needless to say, we have very many challenges ahead of us in trying to understanding the emergence and nature of sonic images in our minds, such as the interactions of sound and body movement as neurocognitive, behavioural and musical-aesthetic phenomena, and such as the temporal re-coding of continuous sound and body movement into more stable images in our minds. Yet from what we know now, it seems reasonable to suggest that images of sound-related actions may be a significant component of sonic images. Images of sound-related actions can trigger sonic images in our minds and can have great exploratory potential. Action imagery in musical imagery can actually be seen as a strategy to implement Schaeffer's method for exploring sonic objects, meaning progressively discovering and becoming aware of more and more sonic features by actively tracing their shapes and their evolution. This also means thinking features in a top-down manner, starting out from low-acuity sketches of overall shapes of the sonic objects (as in Schaeffer's typology), and gradually differentiating more and more features and sub-features (as in Schaeffer's morphology) by actively tracing them, as well as (as a long-term goal) correlating these actively traced features with the acoustic features of the sonic objects.

\section{REFERENCES}

Bangert, M., and Altenmüller, E. O. 2003. Mapping Perception to Action in Piano Practice: A Longitudinal DC-EEG Study. BMC Neuroscience 4: 26.

Berthoz, A. 1997. Le sens du mouvement. Paris: Odile Jacob. Chion, M. 1983. Guide des objets sonores. Paris: INA/GRM Buchet/Chastel.

Eitan, Z., and Granot, R. Y. 2006. How Music Moves: Musical Parameters and Listeners' Images of Motion. Music Perception 23(3): 221-47.
Eitan, Z., and Granot, R. Y. 2008. Growing Oranges on Mozart's Apple Tree: 'Inner Form' and Aesthetic Judgment. Music Perception 25(5): 397-417.

Elliott, D., Helsen, W., and Chua, R. 2001. A Century Later: Woodworth's (1899) Two-Component Model of GoalDirected Aiming. Psychological Bulletin 127(3): 342-57.

Galantucci, B., Fowler, C. A., and Turvey, M. T. 2006. The Motor Theory of Speech Perception Reviewed. Psychonomic Bulletin \& Review 13(3): 361-77.

Gallese, V., and Metzinger, T. 2003. Motor Ontology: The Representational Reality Of Goals, Actions And Selves. Philosophical Psychology 16(3): 365-88.

Gjerdingen, R. O., and Perrott, D. 2008. Scanning the Dial: The Rapid Recognition of Music Genres. Journal of New Music Research 37(2): 93-100.

Godøy, R. I. 1997. Formalization and Epistemology. Oslo: Scandinavian University Press.

Godøy, R. I. 2003. Motor-mimetic Music Cognition. Leonardo 36(4): 317-9.

Godøy, R. I. 2006. Gestural-Sonorous Objects: Embodied Extensions of Schaeffer's Conceptual Apparatus. Organised Sound 11(2): 149-57.

Godøy, R. I. 2008. Reflections on Chunking in Music. In A. Schneider (ed.) Systematic and Comparative Musicology: Concepts, Methods, Findings. Hamburger Jahrbuch für Musikwissenschaft, Band 24. Peter Lang: Frankfurt am Main, 117-32.

Godøy, R. I. 2010a. Gestural Affordances of Musical Sound. In R. I. Godøy and M. Leman (eds.) Musical Gestures. Sound, Movement, and Meaning. New York: Routledge, 105-27.

Godøy, R. I. 2010b. Thinking Now-Points in MusicRelated Movement. In R. Bader, C. Neuhaus and U. Morgenstern (eds.) Concepts, Experiments, and Fieldwork. Studies in Systematic Musicology and Ethnomusicology. Peter Lang: Frankfurt am Main, 241-58.

Godøy, R. I., and Jørgensen, H. (eds.) 2001. Musical Imagery. Lisse: Swets \& Zeitlinger.

Godøy, R. I., and Leman, M. (eds.) 2010. Musical Gestures. Sound, Movement, and Meaning. New York: Routledge.

Godøy, R. I., Haga, E., and Jensenius, A. 2006a. Playing 'Air Instruments': Mimicry of Sound-producing Gestures by Novices and Experts. In S. Gibet, N. Courty and J.-F. Kamp (eds.) Gesture in Human-Computer Interaction and Simulation: 6th International Gesture Workshop, LNAI 3881. Berlin: Springer-Verlag, 256-67.

Godøy, R. I., Haga, E., and Jensenius, A. R. 2006 b. Exploring Music-Related Gestures by Sound-Tracing: A Preliminary Study. In K. Ng (ed.) Proceedings of the COST287-ConGAS 2nd International Symposium on Gesture Interfaces for Multimedia Systems. Leeds University: Leeds, 27-33.

Godøy, R. I., Jensenius, A. R., and Nymoen, K. 2009. Chunking by Coarticulation in Music-related Gestures. Paper presented at The 8th International Gesture Workshop, Bielefeld, 25-27 February 2009.

Haga, E. 2008. Correspondences between Music and Body Movement. Doctoral dissertation, University of Oslo.

Haken, H., Kelso, J. A. S., and Bunz, H. 1985. A Theoretical Model of Phase Transitions in Human Hand Movements. Biological Cybernetics 51(5): 347-56. 
Haslinger, B., Erhard, P., Altenmüller, E., Schroeder, U., Boecker, H., and Ceballos-Baumann, A. O. 2005. Transmodal Sensorimotor Networks during Action Observation in Professional Pianists. Journal of Cognitive Neuroscience 17: 282-93.

Haueisen, J., and Knösche, T. R. 2001. Involuntary Motor Activity in Pianists Evoked by Music Perception. Journal of Cognitive Neuroscience 13(6): 786-92.

Hunt, A., Wanderley, M., and Paradis, M. 2003. The Importance of Parameter Mapping in Electronic Instrument Design. Journal of New Music Research 32(4): 429-40.

Husserl, E. 1991. On the Phenomenology of the Consciousness of Internal Time, 1893-1917. English translation by John Barnett Brough. Dordrecht, Boston and London: Kluwer Academic Publishers.

Kelso, J. A. S., Fuchs, A., Lancaster, R., Holroyd, T., Cheyne, D., and Weinberg, H. 1998. Dynamic Cortical Activity in the Human Brain Reveals Motor Equivalence. Nature 392(23): 814-8.

Kendall, R. A., and Carterette, E. C. 1993. Identification and blend of timbres as a basis for orchestration. Contemporary Music Review 9(1\&2): 51-67.

Kohler, E., Keysers, C., Umiltà, M. A., Fogassi, L., Gallese, V., and Rizzolatti, G. 2002. Hearing sounds, Understanding Actions: Action Representation in Mirror Neurons. Science 297: 846-8.

Kosslyn, S. 1994. Image and Brain: The Resolution of the Imagery Debate. Cambridge, MA: The MIT Press.

Lashley, K. S. 1951. The Problem of Serial Order in Behavior. In L. A. Jeffress (ed.) Cerebral Mechanisms in Behavior. New York: Wiley, 112-31.

Leman, M. 2008. Embodied Music Cognition and Mediation Technology. Cambridge, MA: The MIT Press.

Michon, J. 1978. The Making of the Present: A Tutorial Review. In J. Requin (ed.) Attention and Performance VII. Hillsdale, NJ: Erlbaum, 89-111.
Miller, G. A. 1956. The Magic Number Seven, Plus or Minus Two: Some Limits on our Capacity for Processing Information. Psychological Review 63: 81-97.

Moore, B. C. J. (ed.) 1995. Hearing. San Diego, CA: Academic Press.

Porcello, T. 2004. Speaking of Sound: Language and the Professionalization of Sound-Recording Engineers. Social Studies of Science 34(5): 733-58.

Reisberg, D. (ed.) 1992. Auditory Imagery. Hillsdale, NJ: Erlbaum.

Rosenbaum, D., Cohen, R. G., Jax, S. A., Weiss, D. J., and van der Wel, R. 2007. The Problem of Serial Order in Behavior: Lashley's Legacy. Human Movement Science 26(4): 525-54.

Schaeffer, P. 1966. Traité des objets musicaux. Paris: Éditions du Seuil.

Schaeffer, P. (with sound examples by Reibel, G., and Ferreyra, B.) 1998. Solfège de l'objet sonore. Paris: INA/GRM.

Schleidt, M., and Kien, J. 1997. Segmentation in Behavior and What it Can Tell us about Brain Function. Human Nature 8(1): 77-111.

Schneider, A., and Godøy, R. I. 2001. Perspectives and Challenges of Musical Imagery. In R. I. Godøy and H. Jørgensen (eds.) Musical Imagery. Lisse: Swets \& Zeitlinger, 5-26.

Snyder, B. 2000. Music and Memory: An Introduction. Cambridge, MA: The MIT Press.

Stern, W. 2004. The Present Moment in Psychotherapy and Everyday Life. New York: W.W. Norton.

Wanderley, M., and Battier, M. (eds.) 2000. Trends in Gestural Control of Music. Paris: Ircam.

Wilson, M., and Knoblich, G. 2005. The Case for Motor Involvement in Perceiving Conspecifics. Psychological Bulletin 131(3): 460-73.

Xenakis, I. 1992. Formalized Music. Stuyvesant, NY: Pendragon Press.

Zatorre, R. J., and Halpern, A. R. 2005. Mental Concerts: Musical Imagery and Auditory Cortex. Neuron 47: 9-12. 